\title{
UCRL-TR-220486
}

LAW RENCE LIVERMORE N A T IO N A L LABORATORY
Modeling the QCD Equation of State in Relativistic Heavy Ion Collisions on BlueGene/L

R. Soltz, J. Grady, E. P. Hartouni, R. Gupta, I. Vitev, E. Mottola, P. Petreczky, F. Karsch, N. Christ, R. Mawhinney, S. Bass, B. Mueller, P. Vranas, L. Levkova, D. Molnar, D. Teaney, C. De Tar, D. Toussaint, R. Sugar

April 10, 2006 
This document was prepared as an account of work sponsored by an agency of the United States Government. Neither the United States Government nor the University of California nor any of their employees, makes any warranty, express or implied, or assumes any legal liability or responsibility for the accuracy, completeness, or usefulness of any information, apparatus, product, or process disclosed, or represents that its use would not infringe privately owned rights. Reference herein to any specific commercial product, process, or service by trade name, trademark, manufacturer, or otherwise, does not necessarily constitute or imply its endorsement, recommendation, or favoring by the United States Government or the University of California. The views and opinions of authors expressed herein do not necessarily state or reflect those of the United States Government or the University of California, and shall not be used for advertising or product endorsement purposes.

This work was performed under the auspices of the U.S. Department of Energy by University of California, Lawrence Livermore National Laboratory under Contract W-7405-Eng-48. 


\section{Modeling the QCD Equation of State in Relativistic Heavy Ion Collisions on BlueGene/L}

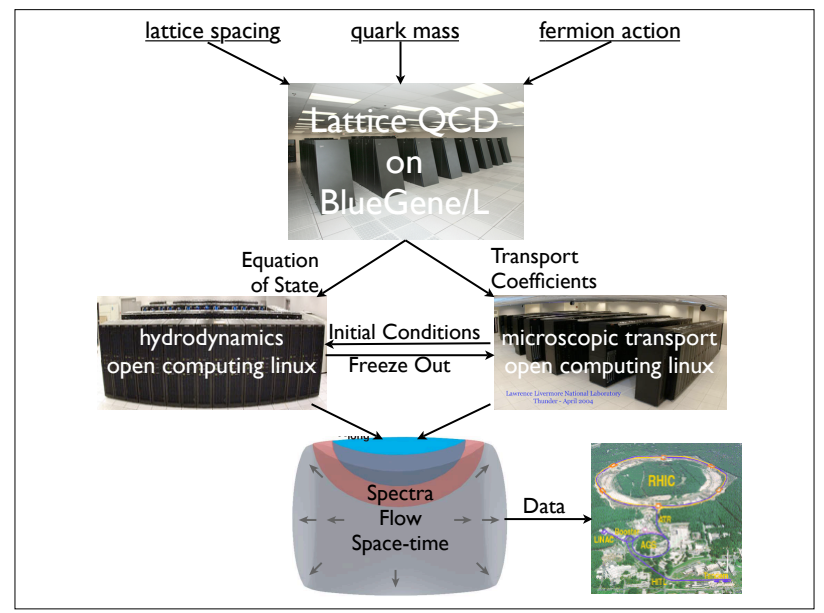

May 31, 2006

R. Soltz (PI), J. Grandy and E.P. Hartouni, LLNL R. Gupta (PI), I. Vitev and E. Mottola, LANL P. Petreczky and F. Karsch, BNL

N. Christ and R. Mawhinney, Columbia University

S. Bass and B. Mueller, Duke University

P. Vranas, IBM Watson Research

L. Levkova, Indiana University

D. Molnar, Purdue University

D. Teaney, SUNY Stony Brook

C. De Tar, University of Utah

D. Toussaint, University of Arizona

R. Sugar, UC Santa Barbara 


\begin{abstract}
On 9,10 Feb 2006 a workshop was held at LLNL to discuss how a $10 \%$ allocation of the ASC BG/L supercomputer performing a finite temperature Lattice QCD (LQCD) calculation of the equation of state and non-equilibrium properties of the quark-gluon state of matter could lead to a breakthrough in our understanding of recent data from the Relativistic Heavy Ion Collider at Brookhaven National Lab. From this meeting and subsequent discussions we present a detailed plan for this calculation, including mechanisms for working in a secure computing environment and inserting the resulting equation of state into hydrodynamic transport models that will be compared directly to the RHIC data. We discuss expected benefits for DOE Office of Science research programs within the context of the NNSA mission.
\end{abstract}

\title{
Contents
}

1 Executive Summary 3

2 Background 4

2.1 RHIC Results . . . . . . . . . . . . . . . . . . . . . . 4

2.2 LQCD Thermodynamics . . . . . . . . . . . . . . . 4

2.3 NNSA Resources . . . . . . . . . . . . . . . . 7

3 Research Proposal $\quad 7$

3.1 LQCD . . . . . . . . . . . . . . . . . . . . . . . . . . . 9

3.2 Hydrodynamics and Microscopic Transport . . . . . . . . . . . . . . . 10

3.3 Data Comparisons ................... . . 11

4 Impact 11

4.1 DOE Office of Science . . . . . . . . . . . . . . . . . . . 11

4.2 NNSA . . . . . . . . . . . . . . . . . . . . . . . 12

4.3 Long Range Plan . . . . . . . . . . . . . . . . . . . . . 13

$\begin{array}{lll}5 & \text { Milestones } & 14\end{array}$

6 Organization $\quad 14$

A Estimates for Improved Staggered Fermions $\quad 16$

$\begin{array}{ll}\text { B Estimates for Domain Wall Fermions } & 17\end{array}$ 


\section{Executive Summary}

The Relativistic Heavy Ion Collider (RHIC) has recently produced a wealth of new data that provide compelling evidence for the creation of a quark gluon plasma in the laboratory. However, the determination of its properties requires a comprehensive modeling effort that is just getting underway. This effort has three components: the determination of the equation of state from first principles calculations of the thermodynamic properties of QCD on a Lattice, the development of properly constrained 3D hydrodynamics codes to compare to data, and the development of new theoretical and computational methods to determine the non-equilibrium transport properties of the quark-gluon plasma (QGP).

Current efforts to calculate the equation of state in Lattice QCD with reliable extrapolations to the continuum limit will take several years using currently available resources. We show that this calculation can be performed in $~ 1$ year with a $10 \%$ allocation of the NNSA BlueGene/L supercomputer. LLNL and LANL researchers will carry out this calculation in the secure computing environment and the resulting equation of state will be transferred to the open computing clusters for use in hydrodynamic codes. External collaborations with the Lattice QCD and RHIC theory communities will contribute to code development and validation. The presence of active experimental groups at both LLNL and LANL will ensure that comparisons to data remain a strong focus of this effort. These collaborations will be maintained through regular teleconferences and meetings at LLNL and LANL. A continuation of the current series of workshops on strongly coupled plasmas and RHIC physics at BNL, LLNL, and LANL will play a vital role in advancing the longer term goals of this proposal.

This multi-step modeling program will significantly advance our understanding of the current RHIC data sets and provide guidance to future experiments - - colliding beams of heavy ions at higher energies at the LHC and at higher luminosities at RHIC II. This program also serves to bring together the scientific communities at the NNSA and at RHIC at a time when both fields are converging upon the study of plasmas in the strong coupling regime. Such cross-fertilization stands to benefit both communities, and enhance the recruiting of NNSA scientists when science plays an ever increasing role in our national security mission. A modest allocation of the $\mathrm{BG} / \mathrm{L}$ computer at this time can have far reaching implications for scientific payoff from the DOE investment in RHIC and the NNSA mission in national security. 


\section{Background}

\section{$2.1 \quad$ RHIC Results}

Recent results from the Relativistic Heavy Ion Collider (RHIC) have provided compelling evidence for the creation of a new state of matter that is best described as a strongly coupled quark-gluon plasma (QGP) with features that are suggestive of a low viscosity nearly perfect fluid [1]. The evidence for this comes from jet-quenching, the suppression of high transverse momentum particles expected in the direction opposite to an observed jet; and soft (low momentum) physics observables such as the anisotropy of low transverse momentum particles that is qualitatively reproduced by hydrodynamic transport models.

Current hydrodynamic models are only partially successful in explaining the complete set of soft physics variables. A consistent description of the transverse momentum distributions, their anisotropy, and the space-time evolution has not yet been achieved, and the prescription for validating models with data is still in its early stages. Instead of starting from a set of well defined or calculated initial conditions, the initial hydrodynamic conditions are tuned to match results. The freeze-out condition is abrupt, i.e. the transition from short mean free path to free streaming particles is instantaneous, and the equation of state is taken to be a simplified first order phase transition from Stefan-Boltzmann gas of quarks and gluons to hadrons. To fully mine the potential of the RHIC data sets requires a comprehensive modeling effort in which the initial and final state conditions of the hydrodynamic transport are better constrained via microscopic transport calculations and the input equation of state is calculated rather than assumed.

Recent work to couple microscopic hadronic transport codes to the end stages of a hydrodynamic calculation have shown some promise $[2,3]$. These efforts are soon to become more cohesive and organized under the "RHIC Transport Initiative", a SciDAC proposal designed to address the current gaps in the application of transport calculations to the RHIC data, such as the need for improved constraints on the initial conditions, the use of an accurate equation of state, and a self-consistent treatment of the freeze-out conditions.

\subsection{LQCD Thermodynamics}

Lattice QCD is widely accepted as the most promising method for calculating the thermodynamics of QCD at finite temperature. The technique, pioneered by Wilson [4] and Creutz [5], replaces the space-time continuum with a 4-dimensional Euclidean lattice on which the QCD partition function can be numerically integrated. 
The primary inputs to a calculation are the number of space-time points on the lattice, the quark masses, the temperature, and the choice of the fermion action (to minimize discretization errors for fixed lattice spacing) used to calculate the partition function. Thus, lattice simulations provide a laboratory for studying nonperturbative behavior of QCD from first principles, i.e. we can study the dependence of the theory on the temperature and the number and masses of quark flavors as these are tunable input parameters.

In a typical calculation the equation of state is calculated at fixed physical temperature, volume and quark masses at a number of values of the lattice spacing $a$. The final equation of state is obtained by extrapolating first in volume $V \rightarrow \infty$ and then to the continuum limit $a \rightarrow 0$. A good choice of the action and the ability to make reliable extrapolation to the contiuuum limit are at the heart of obtaining high precision results.

Lattice QCD calculations are among the most computationally demanding and Lattice QCD community has consistently pushed the state-of-the-art in high performance computing and numerical algorithm development. These demands are especially evident in the approach to the continuum limit. The computational cost for an LQCD calculation varies with quark mass $m_{q}$, lattice spacing $a$, and volume $V$ as $V a^{-7} m_{q}^{-2.5}$ [6]. The volume and temperature are related to the lattice spacing and number of space-time points through the number of lattice steps in space, $N_{\sigma}$, and time, $N_{\tau}$. For thermodynamic calculations, past experience shows that reliable results are obtained for an aspect ratio $N_{\sigma} / N_{\tau}$ of three to four.

The left panel of Fig. 1 shows the most recent results for equation of state with statistical errors for two variations of improved staggered fermion action. The AsqTad action results are for $N_{\tau}=4,6$ and light quark masses corresponding to a pion mass that is twice its physical value. For the p4fat3 action with $N_{\tau}=4$ the pion mass is approximately 4 times its physical value. Additional calculations with the p4fat3 action for $N_{f}=2+1$ and $N_{\tau}=4,6$ are currently underway on the QCDOC. All quantities in the plot have been scaled by the critical temperature. These simulations lead to current estimates with approximately $10 \%$ uncertainty which is dominated by the continuum correction. Confidence in the continuum extrapolation is largely based upon perturbative calculations [7], but an accurate error estimate for the continuum extrapolation for either choice of action requires at least three points, $N_{\tau}=4,6$ and 8. The CPU requirements for $N_{\tau}=8$ calculation exceed what can be achieved with current resources; this forms the crux of our research plan.

The right panel of Fig. 1 summarizes recent calculations of the critical temperature using different improved and unimproved fermion actions. The current best estimate of the cricital temperature for $N_{f}=2$ and $N_{f}=2+1$ is $170 \pm 10 \mathrm{MeV}[10,11]$. 

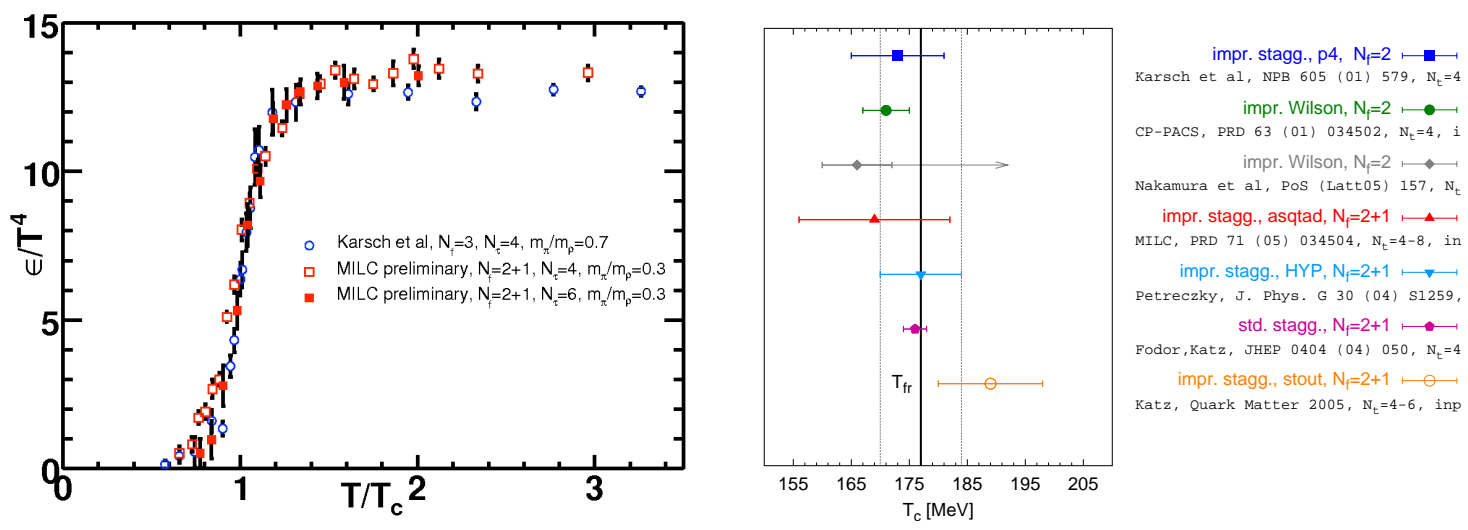

Figure 1: Summary of current equation of state results for improved staggered fermion actions $[8,9]$

This seemingly small uncertainty in the critical temperature corresponds to a $\sim 50 \%$ error in the value of the critical energy density which can have significant implications for the RHIC space-time measurements [2]. The current research plan on the QCDOC aims to reduce this to $20 \%$ uncertainty.

Current simulations of QCD thermodynamics are mostly based on using improved staggered fermions (AsqTad and p4fat3 are two versions of these). These lattice formulations have the advantage of computational speed and retain enough of the continuum chiral symmetry which is necessary for obtaining reliable estimates in the small up and down quark mass limit. Staggered fermions, however, retain chiral symmetry at the cost of flavor doubling - - the discretized theory introduces four copies, called "taste", of each flavor. On the lattice this taste symmetry is broken and introduces addition systematic errors. Alternative formulations such as domain wall fermions (DWF) preserve this symmetry without introducing doubling, and can provide an important check on systematic errors associated with staggered fermion actions. However, calculations of thermodynamic quantities using DWF require considerable additional computational expense [12]. We show that if sufficient resources were available we can carry out a calculation of $T_{c}$ with DWF and $N_{\tau}=8$ that would provide a breakthrough in lattice thermodynamics and an important check on the staggered results. 


\subsection{NNSA Resources}

The NNSA's Advanced Simulation and Computing (ASC) program at LLNL is home to the 360 Teraflop BlueGene/L, the world's fastest supercomputer in 2005 [13]. The IBM BG/L computer is similar in architecture to the QCDOC machine, and LQCD codes have already been shown to run on BG/L computers at IBM's Watson Research Laboratory [14] and also on the LLNL BG/L during its installation tests. The size and scope of the LLNL BG/L supercomputer is demonstrated by the fact that a $10 \%$ allocation specifically targeted towards calculating the QCD equation of state would have an enormous impact on the field of relativistic heavy ion physics. In addition to the science impact, it is important to note that scientists at RHIC and the NNSA have a converging interest in understanding the physics of strongly coupled plasmas, and that the subsequent modeling efforts will provide additional opportunities for cross-fertilization of people and ideas between the Office of Science and the NNSA laboratories. The broader science, recruiting and retention issues are addressed further in Section 4.

\section{Research Proposal}

We propose first to perform a series of lattice QCD calculations using a $10 \%$ allocation of the BlueGene/L supercomputer that will allow for the first full QCD continuum extrapolation of the equation of state with improved staggered fermion actions using $N_{\tau}=4,6,8$ lattices. In addition, we will explore the systematic errors in the staggered fermion approach with the first high accuracy domain wall fermion calculation of the critical temperature. This set of calculations will significantly extend current work by the MILC and RBC-Bielefeld collaborations to produce a definitive equation of state at zero baryon chemical potential that would be suitable for insertion into existing perfect fluid hydrodynamic codes as well as codes to be developed by the RHIC transport institute. Our goal is to create a realistic model in which the initial conditions and final state freeze-out are constrained with partonic and hadronic microscopic transport respectively, with the ultimate goal of comparing directly to the RHIC data sets. With the inclusion of viscous effects (a stated goal of the RHIC Transport Institute) the improved heavy quark energy loss and transverse momentum distributions of elliptic flow anticipated by the forward vertex detector upgrade of the PHENIX experiment (funded in part by a LANL LDRD DR grant) will form an important part of the improved RHIC data to which our modeling effort will be directed.

This research plan will be conducted by LLNL and LANL scientists in close 


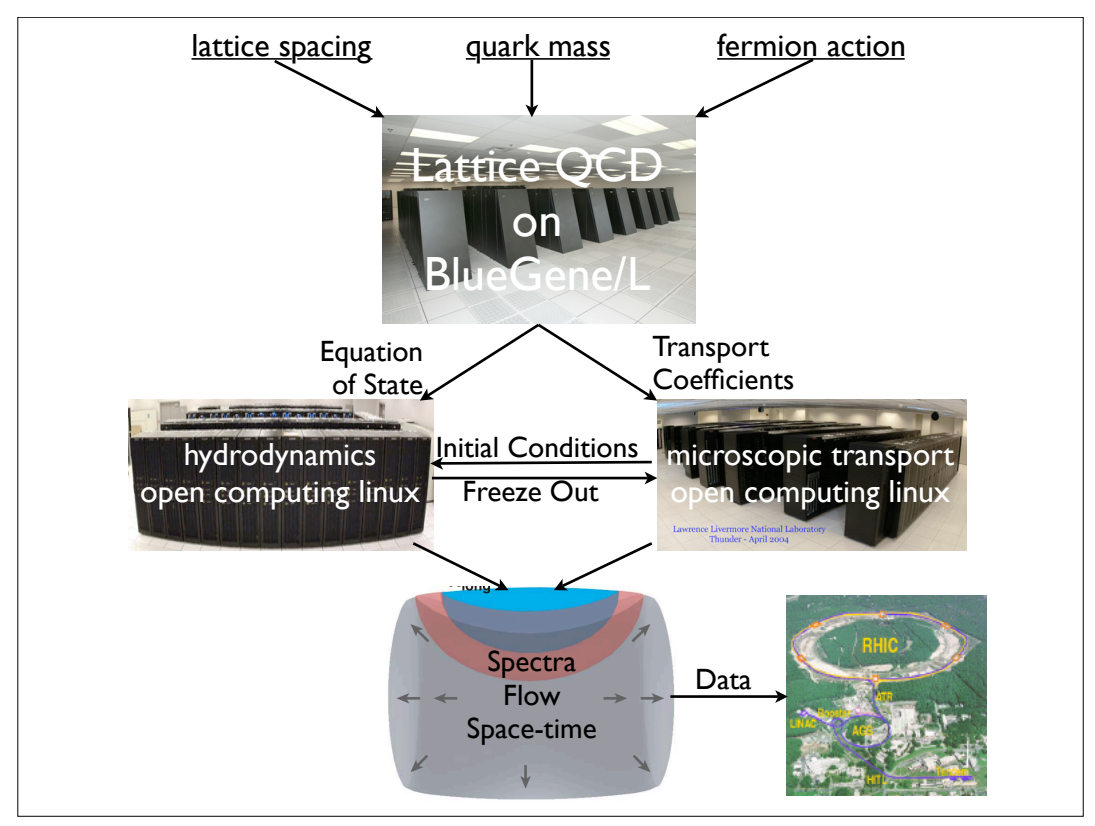

Figure 2: Schematic flow diagram for LQCD, hydrodynamics, microscopic transport, and comparison to RHIC data.

collaboration with both the LQCD and RHIC phenomenology communities. As such, this plan leverages existing NNSA resources to significantly extend and link research activities currently funded by the Office of Science. The overall goal is to use these resources to gain maximal insight into the RHIC data sets.

The overall research program is shown schematically in Fig. 2. The choice of fermion action, lattice size, and quark masses are the essential inputs in LQCD calculations that will be run on BlueGene/L. The calculated equation of state is then fed into the hydrodynamic calculations that are performed on open capacity linux clusters such as MCR and Thunder. Initial conditions to hydrodynamic calculations are provided by partonic microscopic transport and the final state freeze-out of hadrons is fed into a microscopic transport code with hadronic degrees of freedom. In the longer term we will develop codes to measure the spectral and correlation functions necessary to extract the transport coefficients from the improved lattice simulations. This is a challenging problem that will require new theoretical techniques beyond those currently available, but are ultimately necessary for a comprehensive approach 
to the QGP and RHIC data. In the final and most important step, predictions for particle spectra, flow, and space-time evolution will be compared directly to experimental results.

\section{$3.1 \quad$ LQCD}

We plan to perform all Lattice calculations using the Columbia Physics code [15]. These codes will be tested on the open BG/L rack at LLNL or on other BlueGene installations and improvements and bug fixes will be synchronized with our calculations. This code package currently supports the AsqTad, p4fat3, and DWF actions, allowing us to switch quickly and easily between them. We estimate that the full $N_{\tau}=8$ calculation will require, assuming a $20 \%$ efficiency for the LQCD codes on BG/L, 38 TF-yrs (peak) using the p4fat4 action, and 19 TF-yrs with the AsqTad action. Detailed estimates for calculations with improved staggered actions are given in Appendix A. Given a sustained $10 \%$ allocation on the full LLNL BG/L installation this calculation would require just over one year for p4fat3, and approximatly six months for AsqTad. Because the LQCD simulations scale well on BG/L, these calculations could proceed in almost any combination of run allocations with nearly identical efficiency [14].

For a given set of lattice parameters the AsqTad action runs approximately twice as fast as p4fat3, but with possibly larger lattice discretization errors. For this reason, the decision of which improved staggered action to use has been left open and will be determined based on detailed ongoing tests of efficiency and systematic uncertainties. The final decision will depend on which lattice QCD action will lead to the most effective use of $\mathrm{BG} / \mathrm{L}$ resource for improving our understanding of the equation of state and will take into consideration how the BG/L calculation will couple to other ongoing efforts.

A primary consideration for this proposal is how to carry out the calculation in a secure computing environment while maintaining contact with the external community. Access to the machine will be limited to those with security clearances, and the initial team will consist of R. Soltz, R. Gupta, and J. Grandy. In addition to working closely with colleagues at other institutions, we anticipate hiring one or more postdocs and staff with experience in QCD thermodynamics to work at LLNL and LANL and to provide quality assurance and feedback on the output by performing similar tests with the code running on open BG/L systems. All resulting lattice configurations will be stored in the secure HPSS system in Livermore Computing. The lattice data will be analyzed on the secure computing network and reduced to a manageable physics set including associated diagnostics that can be de-classified 
by an Authorized Derivative Classifier (ADC). The equation of state derived from these data can be transferred to the open computing clusters for insertion into hydrodynamic calculations and written up for publication in peer reviewed journals.

\subsection{Hydrodynamics and Microscopic Transport}

For the transport calculations we intend to make maximal use of the codes provided by the RHIC Transport Initiative (RTI) recently proposed to SciDAC by Berndt Mueller and collaborators. RTI is a collaboration of theorists at Duke, MSU, Minnesota, Purdue, and OSU that are working on a standard set of codes and prescriptions for comparing transport models to the RHIC data. The full proposal encompasses a 5-year effort to develop a comprehensive set of transport, benchmark, and visualization tools that will be used to understand the RHIC data. The initial Stage1 deliverables include a 3D relativistic hydrodynamic code in which initial conditions are calculated from a parton cascade and final state freeze-out conditions are determined from a hadronic cascade with a correlation afterburner (a phenomenological incorporation of final state interactions between hadrons) that can reproduce momentum correlations used to extract space-time information in both data and models. It is thus natural to maintain close contact with this collaboration, although it is not necessary to wait for the final release of the Stage- 1 codes. Each of these codes already exists in some form, and we intend to begin working with the authors and to provide feedback while the coding development progresses. In fact, many of the participants are already working on their codes on Livermore Computing clusters in collaboration with members of the LLNL experimental heavy ion group.

While the initial LQCD calculations are running, we intend to begin hydrodynamic calculations using the published equation of state values shown in Fig. 1 using the 3D hydro code under development by Steffen Bass of Duke (to be the nucleus of the RTI effort) for a standard set of initial conditions and without afterburners. Our initial calculations will focus on exploring the systematic errors attributable to uncertainties in the equation of state in energy/momentum and space-time distributions of the pure hydrodynamic models. We will also compare to at least one other hydrodynamic code. One promising candidate is the relativistic version of the FLASH hydrodynamic code developed by the ASC program under the direction of Steve Libby of LLNL. If this code is found to be applicable for heavy ion collisions, it would constitute the first example of a shared resource between the NNSA and RHIC communities.

The second step will be to feed the output of the energy-momentum distributions from the hydrodynamic model into the UrQMD hadronic cascade and correlation 
afterburner, similar to calculations performed by Soff et al. to predict the space-time correlations [2]. This will allow direct comparison to the experimental observables

of spectra, flow, and space-time correlations. The hydrodynamic modeling will be compared to the parton cascade of D. Molnar of Purdue to constrain the initial conditions. These calculations will take place on open computing cluster, and one on which the code authors will have direct access to the code and output. This phase of the research will be conducted in collaboration with the theorists in the RHIC community. When ready, the full equation of state calculated on the lattice will be inserted into the transport models to compare to data.

\subsection{Data Comparisons}

The ultimate goal is to perform a simultaneous comparison of the first principles multi-stage model outlined above to the soft physics signatures which have so far eluded explanation through hydrodynamic calculations with a first order phase transition from an ideal gas of deconfined quarks and gluons. In this respect our approach differs from the proposed RTI that will attempt to back out the equation of state from the data. For this project we will model only in the forward direction, beginning with the equation of state and producing particle spectra, flow, and space-time measurements while propagating associated systematic errors. Both approaches are needed, but our goal is to investigate specifically whether our current understanding of the physics combined with state-of-the-art models and computational resources are adequate for describing the experimental data.

This aspect will also require a close collaboration with the experimental community. In addition to working closely with the experimental groups at LLNL and LANL who are members of the PHENIX experiment, we will enlist the help of collaborators on the other RHIC experiments to insure that experimental acceptances and efficiencies are handled appropriately when comparing to the data.

\section{Impact}

\subsection{DOE Office of Science}

The study of nuclear matter at high energy density is one of the top priorities in the Nuclear Science Advisory Committee (NSAC) long range plan and it has received much attention recently in the popular press due to the wealth of data from RHIC. The overall goal of this proposal is well aligned with this priority. At the conclusion of this project we will have calculated the Equation of State to the highest possible 
accuracy and inserted it into a hydrodynamic calculation suitably constrained by initial conditions and final state freeze-out to predict the hadron spectra, flow, and space-time parameters for $\mathrm{Au}-\mathrm{Au}$ collisions for a range of impact parameters at $s_{N N}=200 \mathrm{GeV}$.

In a few years LHC will begin colliding beams of $\mathrm{Pb}$ at $s_{N N}=5.5 \mathrm{TeV}$, a center of mass energy more than 40 times that of RHIC representing an increase greater than RHIC was with regard to the initial CERN program. These new data may confirm theoretical expectations, or provide new surprises. In either case there is a great advantage to having the theoretical tools proposed here in hand, both for their power to explain the RHIC data and to make predictions for the LHC.

Upgrade plans for RHIC II will occur on a similar timescale. And while many of the decisions on upgrade plans are moving forward based upon experimental considerations and approximate theoretical guidance, the RHIC II program only stands to benefit from having improved theoretical predictions and a procedure for making comparisons to the experimental data. Both the LHC and RHIC II underscore the timeliness of this proposal.

\subsection{NNSA}

The multi-stage modeling required to understand the RHIC data is familiar to many of the physicists at LLNL and LANL engaged in the study of high energy density dense plasmas. Questions regarding the applicability of hydrodynamics and thermalization, and the need to extract dynamics from final state measurements are evident in both fields. However, a stronger connection has recently emerged, in the need to understand strongly coupled plasmas. Two workshops, one at BNL [16] and a second at LLNL [17] have brought these two fields into direct contact and led to many thoughful discussions. This project will serve as a focal point to continue to develop and nuture collaboration between the two communities. This focal point will take the form of a small theory group at LLNL that, in collaboration with LANL, will perform the calculations on $\mathrm{BG} / \mathrm{L}$ and the hydrodynamic and microscopic transport calculations on the open computing clusters at LLNL. We also plan to continue this series of workshops, alternating sites between the three national labs at Livermore, Los Alamos, and Brookhaven.

Perhaps a more important consideration for NNSA is the ability to recruit and retain a strong scientific workforce. The NNSA labs already recruit scientists from a variety of sub-fields in high energy and nuclear physics, but there are relatively few paths of research that allow scientists to explore national security applications while also applying their skills to problems in fundamental research. The need and 
challenge of recruiting excellence from the scientific community is highlighted by the ever increasing role that science plays in our national security. This project brings scientists at LLNL and the RHIC community closer and it does so in a way that is highly visible and beneficial for the NNSA labs.

\subsection{Long Range Plan}

Although the bulk of the proposed research plan has a very specific focus on producing a definitive equation of state for zero net baryon density and incorporating that into hydrodynamic calculations, it establishes a model of cooperation between the Office of Science and NNSA that could be used to achieve additional specific scientific objectives in the future that would benefit both programs.

In tandem with the lattice effort we plan to develop new theoretical techniques based on weak coupling resummation methods familiar in plasma and many-body theory to calculate the non-equilibrium transport coefficients of the QGP state. As the lattice data improve we plan to carry out the first measurements of energymomentum correlation functions necessary to extract the hydrodynamic viscosity, conductivity, and other transport coefficients of the QGP. This has not yet been attempted in a systematic way, and therefore is the most challenging longer term goal of the effort. Yet it holds out the intriguing possibility of determining the nonequilibrium properties of the new state of matter by a comprehensive comparison of first principles approaches, both analytic and lattice based, with RHIC data. The challenge of modeling and measuring the non-equilibrium properties of the QGP is related directly to the predictive modeling of the physics of matter under extreme non-equilibrium conditions which forms the core of the NNSA laboratory mission. Consequently we expect to make use of existing expertise at LANL and LLNL, and to develop new techniques that will be of direct application to physicists working primarily in the DP and NW programs at NNSA laboratories.

The Petascale computing resources that the NNSA will soon have will provide additional opportunities for specific gains in basic science research that would not otherwise be possible. Both Lattice QCD and RHIC phenomenology are computationally intensive fields that can easily benefit from such opportunities, and the growing overlap between the latter and NNSA mission needs highlight the potential future benefit for both. 


\section{Milestones}

Year 1 Install Columbia physics code on BG/L

Perform test runs to diagnose problems and refine timing estimates

Select improved staggered action and begin Equation of State calculation

Insert current equation of state into Duke and FLASH hydrodynamic codes

Perform first comparisons to data

Year 2 Extract equation of state from first LQCD run

Insert improved equation of state into hydrodynamic code

Perform domain wall fermion calculation of critical temperature

Use parton cascade to determine initial conditions for hydro

Publish equation of state results

Publish hdyrodynamic calculations with previous equation of state

Year 3 Perform full hydrodynamic calculation with EOS, and initial and final constraints

Final comparisons to data with full systematic error propagation

Publish domain wall fermion calculation of critical temperature

Publish final hydrodynamic results

\section{Organization}

The success of this project depends upon several collaborations. The most important of these will be the collaboration that we form within the Lattice QCD community that will develop and provide codes, instruction, and feedback on the quality of our results. This will ensure that we make optimal use of BG/L to calculate the continuum extrapolation of the equation of state, and to constrain the systematic errors associated with lattice discretization. It is also important that scientists hired by LLNL and LANL continue to work within the lattice community. Communication will be maintained via teleconferencing on a weekly basis among those actively engaged in calculations, and on a monthly basis with those who will serve in a consulting role. Direct meetings will be held on a quarterly basis between LLNL and LANL participants to which members of the larger collaboration will be invited.

A second collaboration will be formed with those in the RHIC Transport Initiative. Some members of this collaboration have already been granted participating guest status at LLNL and work on code development on the open linux clusters. Our relation to the RTI will be more open, teams will work as computer users rather than co-developers. Therefore communication will not need to be as frequent. Our goals can be met by participating in RTI meetings as needed to learn about code development and provide feedback. 
The last set of collaborations are the experimental collaborations without which this effort would not be possible. We expect to establish formal contact with each of the current RHIC experiments to enlist their help in comparing to data, to insure that such comparisons are performed properly, and to disseminate our model results more quickly among the experimental community.

We view the formation and interaction among these different collaborations as a strength of this proposal, given that our long term goal is to improve scientific interaction among the NNSA and DOE labs. The arrival of BG/L at LLNL, a truly world class computing resource, provides a unique opportunity to leverage NNSA resources to enable the RHIC community to understand the properties of the putative quark gluon plasma at a time when the exploratory measurements are in hand, new experimental programs are on the horizon, and the tools and methods used by both the RHIC and NNSA communities to understand the physics of strongly coupled plasmas are becoming more closely aligned. 


\section{A Estimates for Improved Staggered Fermions}

\begin{tabular}{|l||r|r|}
\hline \multicolumn{1}{|l||}{ Lignt quark mass } & \multicolumn{2}{c|}{$m_{q}=0.1 m_{s}$} \\
\hline Temperature & $\mathrm{T}=0$ & Finite T \\
\hline Lattice sites & $32^{4}$ & $32^{3} \times 8$ \\
\hline Trajectories per $\beta$ & 7,000 & 20,000 \\
\hline$\beta$ steps & 16 & 16 \\
\hline Efficiency Factor & $20 \%$ & $20 \%$ \\
\hline TF-yrs (p4fat3) & $30 \mathrm{TF}-\mathrm{yr}$ & $8 \mathrm{TF}-\mathrm{yr}$ \\
\hline Total (p4fat3) & \multicolumn{3}{|c|}{$38 \mathrm{Tf}-\mathrm{yr}$} \\
\hline Total (AsqTad) & \multicolumn{3}{|c|}{19 Tf-yr } \\
\hline
\end{tabular}

Table 1: Estimates for AsqTad and p4fat3 improved staggered fermion actions. Estimates for p4fat3 action taken from $N_{\tau}=4,6$ runs on QCDOC assuming a $1 / 3.5$ efficiency for 10 of the 16 runs. The AsqTad estimate was obtained assuming a factor of two speedup. Sustained performance estimates are based on an assumed efficiency of $20 \%$ on $\mathrm{BG} / \mathrm{L}$.

The estimates in Table 1 were derived from presentations at the workshop and discussions that followed. The p4fat3 estimates are based on $N_{\tau}=4,6$ runs on QCDOC and the factor of two speedup with the AsqTad action is based on experience running both actions using the Columbia Physics Code. 


\section{B Estimates for Domain Wall Fermions}

Tab. 2 gives an esimate for a critical temperature calculation with an error of about $10 \%$ for $(2+1)$ flavors. Estimates are based on preliminary runs on a $\frac{1}{2} \mathrm{BG} / \mathrm{L}$ rack (512 nodes $=8 \times 8 \times 8$ nodes). The size of the fifth dimension is 32 lattice sites and the fifth dimensional mass is set at the fixed value $m_{0}=1.9$.

\begin{tabular}{|l||r|r|}
\hline Temperature & $\mathrm{T}=0$ & Finite T \\
\hline Lattice sites & $16^{3} \times 32$ & $16^{3} \times 8$ \\
\hline Trajectories/Configurations & 50 & 4000 \\
\hline Propogators/Couplings & 200 & 10 \\
\hline Extra mass runs & 4 & 2 \\
\hline Efficiency Factor & $20 \%$ & $20 \%$ \\
\hline TF-yrs (peak) & 4 TF-yr & 4 TF-yr \\
\hline Total (DWF) & \multicolumn{2}{|c||}{8 Tf-yr } \\
\hline
\end{tabular}

Table 2: Estimates for a Domain Wall Fermion calculation of the critical temperature, for an Iwasaki gauge action with $c_{1}=-0.331$. Approximately an additional $0.5 \mathrm{TF}$ yr for a finite volume systematic error estimate on the critical coupling.

\section{References}

[1] K. Adcox et al. Formation of dense partonic matter in relativistic nucleus nucleus collisions at RHIC: Experimental evaluation by the PHENIX collaboration. Nucl. Phys., A757:184-283, 2005.

[2] Sven Soff, Steffen A. Bass, and Adrian Dumitru. Pion interferometry at rhic: Probing a thermalized quark gluon plasma Phys. Rev. Lett., 86:3981-3984, 2001.

[3] T. Hirano, M. Isse, Y. Nara, A. Ohnishi, and K. Yoshino. Hadron - string cascade versus hydrodynamics in $\mathrm{Cu}+\mathrm{Cu}$ collisions at $\mathrm{s}(\mathrm{nn})^{* *}(1 / 2)=200$-gev. Phys. Rev., C72(041901), 2005.

[4] Kenneth G. Wilson. Confinement of quarks. Phys. Rev., D10:2445-2459, 1974.

[5] M. Creutz. Monte carlo study of quantized SU(2) gauge theory. Phys. Rev., D21:2308-2315, 1980. 
[6] R. Brower et al. Nuclear physics with lattice QCD. http://wwwctp.mit.edu/ñegele/WhitePaper.pdf, 2004.

[7] Urs M. Heller, F. Karsch, and B. Sturm. Improved staggered fermion actions for QCD thermodynamics. Phys. Rev., D60:114502, 1999.

[8] C. Bernard et al. The equation of state for QCD with $2+1$ flavors of quarks. PoS, LAT2005:156, 2005.

[9] F. Karsch, E. Laermann, and A. Peikert. The pressure in 2, 2+1 and 3 flavour QCD. Phys. Lett., B478:447-455, 2000.

[10] C. Bernard et al. QCD thermodynamics with three flavors of improved staggered quarks. Phys. Rev., D71:034504, 2005.

[11] Frithjof Karsch. Lattice QCD at high temperature and density. Lect. Notes Phys., 583:209-249, 2002.

[12] P. Chen et al. The finite temperature QCD phase transition with domain wall fermions. Phys. Rev., D64:014503, 2001.

[13] For top 500 supercomputer list see http://www.top500.org/lists/2005/11/basic.

[14] G. Bhanot, D. Chen, A. Gara, J. Sexton, and P. Vranas. QCD on the BlueGene/L supercomputer. Nucl. Phys. Proc. Suppl., 140:823-825, 2005.

[15] http://phys.columbia.edu/ cqft/physics_sfw/physics_sfw.htm.

[16] Workshop on Strongly Coupled Plasmas held at Brookhaven National Labora-

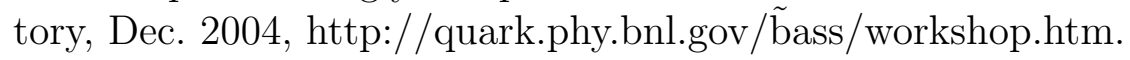

[17] Modeling the QCD Equation of State at RHIC Workshop held at Lawrence Liveremore National Laboratory, Feb. 2006, http://wwwpat.llnl.gov/Conferences/qcdeos06/. 